3. Eine Erweiterung der betriebsverfassungsrechtlichen Kompetenzen und Strukturen durch Tarifvertrag sei ausgeschlossen.

4. Im Vergleich zum geltenden Recht sei der Einfluß auf die leitenden Angestellten nicht verstärkt, sondern eher abgeschwächt worden. Die präzise, allein auf objektive Merkmale gestützte Abgrenzung des leitenden Angestellten lasse eine weitere Erfassung dieses Personenkreises zu, als es nach früherem Recht möglich gewesen sei. Zulässig seien künftig auch Sprecherausschüsse.

5. Tendenzunternehmen genössen größeren Schutz, als nach dem Referentenentwurf vorgesehen. Damit sei die Gewähr gegeben, daß auch künftig eine „faire» Berichterstattung durch Zeitungsverleger gewährleistet sei.

Als Negativpunkte des Gesetzes werden verbucht:

I. Die Vermehrung des Einflusses der Einigungsstellen,

2. die Vorschriften über das Beschwerdeverfahren,

3. die Verstärkung der Rechtsposition des Betriebsrates bei ordentlichen Kündigungen. Prof. ... hat zur Umgebung dieser Vorschriften den Vorschlag gemacht, künflig grundsätzlich außerordentliche Kündigungen auszusprechen, subsidiär zugleich aber eine ordentliche Kündigung nachzuschieben, um die Beschättigungspflicht des Arbeitgebers entfallen zu lassen. Herr Eichler berichtete, daß die Beschäftigungspflicht des Arbeitgebers im Falle eines Widerspruchs gegen eine ordentliche Kündigung durch keinerlei Sanktion abgesichert sei. Lasse der Arbeitgeber den Arbeitnehmer nicht in den Betrieb hinein, so behalte dieser lediglich seinen Lohnanspruch.

4. Die Aufstellung erzwingbarer Personalrichtlinien müsse im Wege der Interpretation entschärft werden, damit der Arbeitgeber eine uneingeschränkte Personalhoheit behalte. Man stehe im Kontakt mit der Deutschen Gesellschaft für Personalführung, die vom Bundesarbeitsministerium einen Gutachtenauftrag zur Konkretisierung der Normen über die quantitative und qualitative Personalplanung erhalten habe.

Herr Eichler behauptete ferner, daß es ganz sicher sei, daß gegen verschiedene Bestimmungen des neuen Gesetzes eine Verfassungsbeschwerde erboben werde, obgleich man hier sich nicht große Chancen auf Erfolg ausrechne.

Angriffe auf die Verfassungsmäßigkeit des neuen Gesetzes schüfen aber im politischen und arbeitsgerichtlichen Raum das Klima für eine restriktive Auslegung der Kompetenzen des neuen Gesetzes. Er bat die anwesenden Wissenschaftler: »im Dienste kühler Vernunfe an solchen Vorhaben mitzuwirken und lud zu einer weiteren Besprechung über das neue Betriebsverfassungsgesetz für den April 1972 ein, wo dann konkrete Vorhaben zur restriktiven Interpretation des neuen Gesetzes besprochen werden könnten. Abgesehen von ... und ... äußerten sich alle Anwesenden, vor allem...... im Sinne einer strikten politischen $A b$ lebnung des neuen kompetenzausweitenden und unternebmerischen Autonomie abbauenden Gesetzes. 


\section{Die staatliche Reglementierung der industriellen Beziehungen in England ${ }^{1}$}

I.

Von dem konservativen Gewerkschaftstheoretiker Goetz Briefs stammt der Begriff der befestigten Gewerkschaft ${ }^{2}$. Mit ihm kennzeichnet er die gewerkschaftlichen Organisationen Westeuropas seit 1914. Dreierlei besagt der Begriff:

erstens genießen die Gewerkschaften die volle Anerkennung durch Gesetzgebung, Arbeitgeber und öffentliche Meinung;

zweitens besitzen sie als Institution eine hohe Festigkeit gegenüber Schwankungen der Wirtschaft;

drittens nehmen sie durch die Zuweisung öffentlicher Funktionen und Verantwortungen den Status einer quasi-öffentlichen Körperschaft an.

Im Zustand der Befestigung und unter Bedingungen der Vollbeschäftigung verfügen die Gewerkschaften und ihre Untergliederungen über eine Verhandlungsmacht, die sie potentiell - gleichfalls nach einem Wort von Briefs - zur "Störungsmacht « ${ }^{3}$ im kapitalistischen System machen.

Die vorherrschende Einkommens- und Vermögensverteilung in kapitalistischen Systemen können die Interessenorganisationen der unterprivilegierten Lohnabhängigen nicht akzeptieren. Versuchen sie jedoch, die Verteilungsverhältnisse über Lohnkämpfe zu verändern, dann können die Unternehmer über Preis- und Investitionsentscheidungen diese Versuche durchkreuzen - allerdings mit der Konsequenz inflationärer Preissteigerungen (Ǔberwälzungsversuch) oder erhöhter Arbeitslosigkeit (Investitionszurückhaltung). Für spätkapitalistische Systeme werden solche Leerlaufmechanismen zur Absicherung der Profitraten immer risikoreicher. Schon eine Arbeitslosenquote von über zwei Prozent kann zu schwer kontrollierbaren politischen Reaktionen (Loyalitätskrise) und zu Wachstumsverlusten führen ${ }^{4}$. Eine Inflationsrate, die nur geringfügig über der der Konkurrenzländer liegt, kann die internationale Wettbewerbsfähigkeit empfindlich treffen und Außenhandelskrisen auslösen.

Das Ziel der Systemstabilität verpflichtet das politische Zentrum in spätkapitalistischen Systemen, Krisen dieser Art zu verhindern. Seine ökonomische Steuerungskapazität ist freilich durch privatwirtschaftliche Eigentumsgarantien begrenzt. Um den Verteilungskampf und seine disruptiven Folgen für Stabilität und Wachstum einzudämmen, wird dem Staat daher die gewerkschaftliche Tarifautonomie zum bevorzugten Aktionsparameter. Damit trifft er allenfalls eine sekundäre Ursache der »säkularen Inflation «; die primäre, welche in der Preissetzungsmacht privater Oligopole lieg $\mathrm{t}^{5}$, bleibt ungeschoren.

II.

Die Eingrenzung der gewerkschaftlichen Autonomie durch den spätkapitalistischen Staat erfolgt in mannigfacher Weise; die beiden extremen Pole sind:

\footnotetext{
1 Der folgende Beitrag schließt thematisch an meinen Aufsatz *Gewerkschaftsgesetzgebung in England an. In ihm habe ich die Grundzüge der britischen industriellen Beziehungen, ihre Entwidklung und Neuregelung unter der Labour-Regierung in den Jahren $1964-69$ dargestellt sowie die ökonomischen Rahmenbedingungen analysiert. Wem die Hinweise darauf im folgenden Beitrag zu kursorisch erscheinen, verweise idh auf den früheren Aufsatz; KJ 3/1969.

2 Vgl. G. Briefs: Zwischen Kapitalismus und Syndikalismus. Die Gewerkschaften am Scheideweg, München I952, S. 87.

3 Vgl. ders. (ed.): Laissez-faire-Pluralismus, Berlin 1966, S. 49.

- Beispiel dafür war die Rezession 1966/67 in der BRD.

3 Vgl. W. Hofmann: Die säkulare Inflation, Berlin 1962.
} 
Kooperation zwischen Staatsapparat und Gewerkschaftsspitzen; Suppression gewerkschaftlicher Aktivitäten durch den Staatsapparat.

Die freiwillige Kooperation zielt darauf ab, den Gewerkschaften eine Selbsteinschränkung ihrer bargaining power abzuverlangen: sie sollen ihre Lohnpolitik nicht nach Machtposition und Marktlage, sondern nach gesamtwirtschaftlichen Stabilitäts- und Wachstumserfordernissen betreiben. Paradigma solcher Kooperation ist die Einkommenspolitik der Bundesregierung seit Begründung der Konzertierten Aktion. Wird die Einkommenspolitik nicht mehr durch »moral persuasion ", sondern durch gesetzliche Maßnahmen erzwungen, wie in den letzten Jahren der Labour-Regierung, dann geht die Kooperation in die Suppression über. Diese zielt auf eine direkte Schwächung der faktischen bargaining power der Gewerkschaften. Beispiel dafür ist der britische Industrial Relations Act, den die konservative Regierungsmehrheit kürzlich verabschiedet hat.

Die konträren Strategien zur Eingrenzung gewerkschaftlicher Autonomie sind an entgegengesetzte Bedingungskomplexe gebunden. Die alternativen Strategien und ihre Bedingungen finden wir fast modellhaft in der Bundesrepublik und in England vor.

Die Kooperation zwischen dem Staatsapparat und den zentralen Gewerkschaftsorganen in der Bundesrepublik beruht auf folgenden Voraussetzungen:

- Garantie und realer Zustand von Vollbeschäftigung,

- reale Einkommensverbesserungen über Produktivitätsfortschritte, nicht durch Umverteilung

- Ansätze zur „Vermögensbildung in Arbeitnehmerhand« (als Ersatz für Umverteilungsziele)

- zentralisierte Verhandlungssysteme,

- geringe Militanz und hohe Folgebereitschaft an der Basis für die Politik der Verbandsspitze ${ }^{6}$.

Die sozialdemokratische Regierung eignet sich zudem besser als Partner der Kooperation als eine konservative Regierung, weil die Gewerkschaften zur Sozialdemokratie in einem traditionellen Loyalitätsverhältnis stehen.

Die Suppression der gewerkschaftlichen Autonomie erfolgt in Großbritannien vor dem Hintergrund entgegengesetzter Bedingungen:

- eine im Verhältnis zur erfahrenen Vollbeschäftigung hohe Arbeitslosigkeit,

- stagnierende oder gar sinkende Realeinkommen,

- fehlende Ansätze zu einer wirtschaftsfriedlichen Vermögenspolitik, - fragmentarisierte und konfligierende Verhandlungssysteme,

- militante Basisbewegungen und Gewerkschaften.

Úberdies befindet sich die konservative Partei an der Regierung, die nicht mit der Loyalität und Kooperationsbereitschaft der Gewerkschaften rechnen kann.

III.

In einem früheren Aufsatz ${ }^{7}$ habe ich auf die strukturellen Ursachen der britischen Wirtschaftskrise hingewiesen. Die ökonomischen Indikatoren ${ }^{8}$ signalisieren seither eine fortschreitende Verschlechterung.

Produktion: Von allen EWG- und EFTA-Staaten hat Großbritannien die geringsten Wachstumsraten. Sie lagen in den sechziger Jahren im Durchschnitt bei

- Für die BRD läßt sich seit den Septemberstreiks 1969 beobachten, daß die angeführten Bedingungen sich verschlechtern bzw. für eine Kooperation nicht mehr ausreichen.

7 s. Anm. I.

8 Die Daten stammen aus folgenden Quellen: Economic Progress Report (monatliche Informationsblätter des Schatzamtes), British Journal of Industrial Relations, London, Jge. 1970 und $x 97$. 
3 Prozent. In den beiden letzten Jahren I 969 und 1970 sind sie unter 2 Prozent gefallen.

Preise: Die Inflationsraten zählen hingegen zu den höchsten in den europäischen Ländern. In den letzten drei Jahren sind die Lebenshaltungskosten progressiv gestiegen: I969 um 5,5 Prozent, 1970 um 8,5 Prozent; gegenwärtig liegt die Jahresrate schon über Io Prozent.

Löbne: Auch die Löhne weisen eine Tendenz progressiver Steigerung aus. 1969 stiegen die Stundenlöhne um 5,9 Prozent, I 970 um I 3,7 Prozent; im I. Quartal 197 I lag die Jahresrate bei I6 Prozent.

Profite: Der Anteil der Profite am Nationaleinkommen (Gewinnquote) ist seit Mitte der sechziger Jahre stetig gefallen.

Beschäftigung: In den vergangenen drei Jahren war die Arbeitslosenquote höher als 2 Prozent; 1971 stieg sie auf 3 Prozent an und nähert sich gegenwärtig einer Quote von 4 Prozent.

Arbeitskämpfe: Am dramatischsten spiegelt sich die Entwicklung in der Streikstatistik wider. I 968 gingen 4,7 Mill. Arbeitstage durch Streiks verloren, I 969 waren es 6,7 Mill. Arbeitstage, 1970 schon I0,9 Mill. Arbeitstage. Im I. Quartal I971 gingen bereits mehr Arbeitstage durch Streiks verloren als im gesamten Jahr 1970 (9,5 Mill. Arbeitstage durch wirtschaftliche und 2,5 Mill. Arbeitstage durch politische Streiks).

Aus diesen Daten läßt sich ohne Mühe die soziale und ökonomische Krise Großbritanniens rekonstruieren. Stagnation und Inflation, in der neokeynesianischen Diskussion bisher als alternative Zustände angesehen, sind in Großbritannien zur Stagflation verschmolzen. Gegen diesen Krisentypus erweisen sich die herkömmlichen wirtschaftspolitischen Rezepte als wirkungslos. Das Zusammentreffen von schrumpfenden Wachstumsraten und steigender Arbeitslosigkeit mit progressiv ansteigenden Löhnen und Preisen spricht ökonomischen Lehrsätzen Hohn ${ }^{8}$.

IV.

Die entscheidende Variable in dem Datengeflecht ist offenbar die Militanz der Lohnabhängigen. Sie setzen höhere Löhne durch, ohne sich mit den schmalen Zuwächsen des nationalen Kuchens abspeisen zu lassen. Zum größeren Teil werden die Lohnerhöhungen über Preiserhöhungen abgewälzt; wo dies die Marktsituation nicht zuläßt, müssen sie aus Profiten finanziert werden. Daraus resultiert die Beschleunigung der inflationären Tendenzen, der Fall der Profitraten und, weil der Widerstand der Unternehmer wächst, die rapide Zunahme der Arbeitskämpfe.

$\mathrm{Da}$ die militanten Lohnkämpfe die strukturelle Krise des Systems verschärfen, liegt es nahe zu folgern, daß ein Wirtschaftssystem, dessen Wachstumsraten kaum Lohnerhöhungsspielräume läßt, vor den britischen Arbeitern seine Legitimität verloren hat. Dies müßte freilich als antikapitalistisches Bewußtsein manifest werden. Indessen ist trotz anhaltender und sich verschärfender Wirtschaftskrise keine bedeutende Zunahme antikapitalistischer Bewegungen konstatierbar. (Den Mitgliederzuwächsen trotzkistischer Organisationen stehen Mitgliederverluste

- Der britische Okonom F. W. Paish hat vor einigen Jahren auf der Grundlage der PhillipsKurve errectnet, daß bei einer Arbeitslosigkeit von etwas über 2 Prozent die Lohnsteigerungsraten nicht über den Steigerungsraten des Sozialprodukts liegen würden (s. Policy for Incomes, London 1967 , S. 2 f.). Heute, bei Arbeitslosenraten von über 3 und Inflationsraten von über ro Prozent verspricht sich der konservative $\rightarrow$ Daily Telegraph diese Wirkung bei einer Arbeitslosenquote von 4,5 Prozent. 
der kommunistischen Partei gegenüber. Bei den Massen wächst allenfalls die Unzufriedenheit mit den Tories.)

Der Widerspruch zwischen den objektiven Wirkungen der Lohnkämpfe und dem subjektiven Bewußtsein davon hat eine Ursache in der Fragmentarisierung des colletive bargaining, das militante Basisbewegungen begünstigt, aber ein Bewußtsein über makroökonomische Auswirkungen kaum aufkommen läßt.

Die Königliche Kommission über Gewerkschaften und Arbeitgeberverbände hat in ihrem 1968 vorgelegten Untersuchungsbericht ${ }^{10}$ von zwei konfligierenden Systemen kollektiver Regelungen als dem Hauptdefekt der britischen industrial relations gesprochen. Das formale System, zentralisiert auf der Ebene von Industriezweigen, wird getragen von offiziellen Beratungs- und Verhandlungskommissionen der Tarifparteien, die in schriftlichen Abkommen Minimallöhne, Standardbedingungen und formalisierte Konfliktregelungen festlegen. Das informale System besteht aus autonomen, fragmentierten und informellen Verhandlungen auf betrieblicher Ebene (workshop bargaining). Die soziale Macht von Arbeitsgruppen und ihren Vertretern, den shop stewards, ist in der Vollbeschäftigungsphase der Nachkriegsperiode gewachsen, mit ihr der Umfang und die Bedeutung des workshop bargaining. Zentrale offizielle Verhandlungen sind für die Arbeiter immer unwichtiger geworden; ihre Do-it-yourself-Aktionen bringen bessere und schnellere Ergebnisse. Die Lohndrift und die hohe Zahl von inoffiziellen Betriebsstreiks ( 90 Prozent aller Streiks) haben darin ihre Ursachen.

Bislang wurden die Basisaktivitäten durch keine arbeitsrechtlichen Sanktionen eingeschränkt. Selbst die zentralen Abkommen der Tarifparteien konnten in der Regel weder in ihren substantiellen noch ihren prozessualen Bestimmungen verbindliche Normen für die betrieblichen Verhandlungen setzen. Einschneidende Veränderungen ergeben sich für die Betriebsorganisationen nunmehr aus den Bestimmungen des Industrial Relations Act.

V.

Der im letzten Jahr gegen die Opposition der Labour Party verabschiedete Industrial Relations Act bricht radikal mit der Tradition der britischen Industrial Relations. Deren Gestaltung beruhte in der Vergangenheit nahezu ausschließlich auf den freiwilligen Vereinbarungen der sozialen Kontrahenten. Die wenigen arbeitsrechtlichen Bestimmungen dienten der Ergänzung oder Stützung dieses freiwilligen Systems kollektiver Regelungen. Die rechtlichen Privilegien, die den Tarifvertragsparteien gewährt wurden, hatten den Charakter von Immunitäten gegenüber Normen des Zivil- und Strafrechts. Diese Immunitäten werden mit dem Industrial Relations Act in hohem Maße eingeschränkt. Durch das Gesetz wird eine umfassende Arbeitsrechtsgesetzgebung institutionalisiert und die bislang unbekannte Institution des Arbeitgerichts begründet.

Die gravierendsten Veränderungen erfahren die traditionellen Streikrechte und die herkömmlichen Regelungen des collective bargaining.

Nach dem Trades Disputes Act I 906 waren die Gewerkschaften und ihre Beauftragten von der zivilrechtlichen Haftung für alle Schäden, die aus Arbeitskämpfen resultieren, befreit. Die Immunität wird mit dem Industrial Relations Act in folgender Weise eingeschränkt:

I. Die Immunität wird auf registrierte Gewerkschaften begrenzt. Registrieren lassen können sich Gewerkschaften, deren Satzung demokratischen Grundsätzen,

$10 \mathrm{Vgl}$. Royal Commission on Trade Unions and Employers' Association r965-1968: Report (Donovan-Report), London 1968, S. 12-37. 
die von der Regierung festgelegt werden, nicht widerspricht. Damit erhält die Regierung die Möglichkeit, auf die interne Struktur der Gewerkschaften Einfluß zu nehmen.

2. Die Immunität wird auf solche Arbeitskämpfe beschränkt, die nicht unter die Kategorie der »unfair industrial practices f fallen; zu diesen zählen u.a. der closed shop, der Bruch von tariflichen Vereinbarungen, der Sympathiestreik und der inoffizielle Streik.

Nach diesen Bestimmungen kann ein Unternehmer oder Unternehmerverband Gewerkschaften und Individuen auf Schadensersatz verklagen, wenn Arbeitskämpfe von nicht-registrierten Gewerkschaften geführt werden und/oder wenn Arbeitskämpfe sich unter die »unfair industrial practices « rubrizieren lassen. Unbeschränkt haftbar sind nicht-registrierte Gewerkschaften und Individuen, die nicht im Auftrag von registrierten Gewerkschaften handeln. Beschränkt haftbar sind registrierte Gewerkschaften; sie haften je nach Mitgliederzahl. (Gewerkschaften mit mehr als 100000 Mitgliedern haften bis zu einem Betrag von roo 000 Pfund Sterling).

Zwei weitere Bestimmungen schränken die gewerkschaftliche Streikfreiheit ein: die Regierung kann bei Streiks, die einen »nationalen Notstand « herbeiführen können, eine Abkühlungsperiode von 60 Tagen sowie eine Urabstimmung anordnen.

Bisher hatten die Tarifverträge $\mathrm{z}$ wischen Gewerkschaften und Unternehmern bzw. ihren Verbänden rechtlich den Status von Gentlemen's Argreements; sie waren von keiner Seite einklagbar. Mit dem Industrial Relations Act wird dieser Status aufgehoben.

I. Alle Abkommen, ob über substantielle Bestimmungen (Lohn- und Arbeitsbedingungen) oder über Verfahrensregelungen (bei Verhandlungen und Konflikten) sind zukünftig rechtlich bindend und einklagbar, sofern nicht beide Vertragskontrahenten sich auf eine gegenteilige schriftliche Abmachung einigen.

2. Das höchste Arbeitsgericht kann bei industriellen Konflikten über Verfahrensregelungen von der Regierung oder einer der Tarifparteien angerufen werden, um die bestehenden kollektiven Regelungen auf ihre Funktionsfähigkeit zu überprüfen und gegebenenfalls neue Regelungen zu empfehlen; auf Antrag einer der Tarifparteien werden diese Regelungen gesetzlich verbindlich gemacht.

Mit der rechtlichen Beschneidung der traditionellen Streikrechte und den gesetzlich erzwingbaren kollektiven Regelungen wird primär die bargaining power von Betriebsorganisationen und Arbeitsgruppen geschwächt. Halten sie sich bei Kampfmaßnahmen nicht an die Satzungsbestimmungen ihrer Gewerkschaft, streiken sie - wie bisher üblich - inoffiziell, so verlieren sie die gesetzliche Immunität gegenüber Schadensersatzansprüchen. Stehen ihre Kampfmaßnahmen nicht im Einklang mit früher ausgehandelten Verfahrensregelungen, so kann ihnen ein gesetzlich bindendes Verfahren der Konfliktregelung aufgezwungen werden. Die konservative Gewerkschaftsgesetzgebung zielt darüber hinaus auf die Schwächung der gesamten britischen Gewerkschaftsbewegung, die in den letzten Jahren - entgegen ihrer früheren Praxis - eine Reihe offizieller Flächenstreiks geführt hat ${ }^{11}$. Nach dem Vorbild des amerikanischen Taft-Hartley-Gesetzes gibt der Industrial Relations Act der Regierung die Vollmacht in die Hand, durch eine Abkühlungsperiode Streiks größeren Ausmaßes zu unterdrücken oder zu illegalisieren, eine Bestimmung, die selbst das deutsche Arbeitsrecht nicht kennt.

11 So im letzten Jahr der neunwöchige Streik von so ooo Fordarbeitern und der sechswöchige Streik von 200000 Postarbeitern. 
VI.

Der konservative Angriff auf die Rechte der britischen Gewerkschaftsbewegung hat deren massiven Widerstand herausgefordert. Seirdem die ersten Vorlagen des Gesetzes bekannt wurden, haben Demonstrationen und Streiks stattgefunden. Erstmals seit dem Generalstreik 1926 ist es wieder zu politischen Streiks mit hohen Beteiligungen gekommen.

Der erste politische Streik wurde noch vom inoffiziellen Committee for the Defense of Free Trade Unionsm für den 8. Dezember 1970 ausgerufen. Obgleich der Trades Union Congress (TUC), die Dachorganisation der britischen Gewerkschaften, sich gegen den Streikaufruf des von kommunistischen Militanten und shop stewards getragenen Komitees ausgesprochen hatte, traten etwa eine halbe Million Arbeiter in den Ausstand. Am 21. Februar 1971, einem Sonntag, organisierte der TUC in London eine Demonstration, an dem 140000 Gewerkschaftler teilnahmen; nach den Worten einer TUC-Publication war dies "the biggest demonstration of the century - the biggest since the Chartist moved working men to demand the right to vote ${ }^{12}$.

Zwei weitere politische Streiks wurden von den großen Metallarbeitergewerkschaften, denen sich später die Transportarbeitergewerkschaft anschloß, ausgerufen. Am r. März r97I folgten ihrem Streikaufruf I,5 Millionen und am I8. März etwa zwei Millionen Arbeiter.

Mit diesen Aktionen war der Höhepunkt der Bewegung gegen das Gewerkschaftsgesetz erreicht. Denn am I8. März konnte der TUC auf einem Sonderkongreß der Gewerkschaftsexekutiven mit Hilfe der rechten Gewerkschaftsvorstände seine Politik der Non-Cooperation durchsetzen. Den affiliierten Organisationen wurde empfohlen: sich nicht registrieren zu lassen, keine rechtlich bindenden Tarifverträge zu unterzeichnen und die Mitarbeit an offiziellen industriellen Gremien einzustellen. Entgegen der Forderung starker Minderheiten, wurden diese Empfehlungen nicht mit Sanktionen ausgestattet; ihre Mißachtung sollte nicht den Ausschluß aus dem Gesamtverband zur Folge haben. Weitere industrielle Aktionen, sprich Streiks, gegen das geplante Gesetz wurden von der Mehrheit abgelehnt. Damit brach die Protestbewegung so erfolglos zusammen wie seinerzeit die Chartistenbewegung, auf die die TUC-Broschüre so stolz sich berief.

Nach der Verabschiedung des Industrial Relations Act durch das Parlament wurde auf dem im September letzten Jahres veranstalteten Kongreß der britischen Gewerkschaften die Linie des TUC bestätigt. Mehrere Gewerkschaften, so besonders die des öffentlichen Dienstes, haben sich bereits offen für die Registrierung (die mit steuerlichen Vorteilen lockt) ausgesprochen; einige von ihnen würden selbst den Ausschluß aus dem Gesamtverband riskieren. $\mathrm{Daß}$ sie dies nicht zu befürchten brauchen, wissen sie, nachdem ein Antrag dieses Inhalts mit den Stimmen der als links geltenden Vorstände der Transportarbeiter- und Metallarbeitergewerkschaft abgelehnt wurde.

Im Gegensatz zu italienischen Gewerkschaften, die ihren Zielen mit direkten Aktionen zustreben, bleibt die britische Gewerkschaftslinke an die parlamentarische Methode fixiert: Nur noch der Wahlsieg der Labour Party kann das Gesetz rückgängig machen. Mit der Parteilinken ("Tribune Left «) verdrängt sie mit diesem Wunsch die Erfahrung, daß die letzte Labour-Regierung die Gesetzgebung gegen die Gewerkschaftsbewegung eingeleitet hat.

Walther Müller-Jentsch

12 Trades Union Congress: The Great March, London 1971 , S. I. 